Int. J. Number Theory 16 (2020), no. 8, 1833-1858.

\title{
QUADRATIC RESIDUES AND QUARTIC RESIDUES MODULO PRIMES
}

\author{
ZHI-WEI SUN
}

\begin{abstract}
In this paper we study some products related to quadratic residues and quartic residues modulo primes. Let $p$ be an odd prime and let $A$ be any
\end{abstract} integer. We determine completely the product

$$
f_{p}(A):=\prod_{\substack{1 \leqslant i, j \leqslant(p-1) / 2 \\ p \nmid i^{2}-A i j-j^{2}}}\left(i^{2}-A i j-j^{2}\right)
$$

modulo $p$; for example, if $p \equiv 1(\bmod 4)$ then

$$
f_{p}(A) \equiv \begin{cases}-\left(A^{2}+4\right)^{(p-1) / 4}(\bmod p) & \text { if }\left(\frac{A^{2}+4}{p}\right)=1 \\ \left(-A^{2}-4\right)^{(p-1) / 4}(\bmod p) & \text { if }\left(\frac{A^{2}+4}{p}\right)=-1\end{cases}
$$

where $(\dot{p})$ denotes the Legendre symbol. We also determine

$$
\prod_{\substack{i, j=1 \\ p \nmid 2 i^{2}+5 i j+2 j^{2}}}^{(p-1) / 2}\left(2 i^{2}+5 i j+2 j^{2}\right) \text { and } \prod_{\substack{i, j=1 \\ p \nmid 2 i^{2}-5 i j+2 j^{2}}}^{(p-1) / 2}\left(2 i^{2}-5 i j+2 j^{2}\right)
$$

modulo $p$.

\section{INTRODUCTION}

For $n \in \mathbb{Z}^{+}=\{1,2,3, \ldots\}$ and $x=a / b$ with $a, b \in \mathbb{Z}, b \neq 0$ and $\operatorname{gcd}(b, n)=1$, we let $\{x\}_{n}$ denote the unique integer $r \in\{0, \ldots, n-1\}$ with $r \equiv x(\bmod n)$ (i.e., $a \equiv b r(\bmod n))$. The well-known Gauss Lemma (see, e.g., [6, p. 52]) states that for any odd prime $p$ and integer $x \not \equiv 0(\bmod p)$ we have

$$
\left(\frac{x}{p}\right)=(-1)^{\left|\left\{1 \leqslant k<p / 2:\{k x\}_{p}>p / 2\right\}\right|},
$$

where $(\dot{p})$ is the Legendre symbol. This was extended to Jacobi symbols by M. Jenkins [7] in 1867, who showed (by an elementary method) that for any positive odd integer $n$ and integer $x$ with $\operatorname{gcd}(x, n)=1$ we have

$$
\left(\frac{x}{n}\right)=(-1)^{\left|\left\{1 \leqslant k<n / 2:\{k x\}_{n}>n / 2\right\}\right|},
$$

where $(\dot{m})$ is the Jacobi symbol. In the textbook [9, Chapters 11-12], H. Rademacher supplied a proof of Jenkins' result by using subtle properties of quadratic Gauss sums.

Now we present our first new theorem.

Theorem 1.1. Let $n$ be a positive odd integer, and let $x \in \mathbb{Z}$ with $\operatorname{gcd}(x(1-x), n)=$ 1. Then

$$
(-1)^{\left|\left\{1 \leqslant k<n / 2:\{k x\}_{n}>k\right\}\right|}=\left(\frac{2 x(1-x)}{n}\right) \text {. }
$$

Key words and phrases. Quadratic residues, quartic residues, congruences, Lucas sequences. 2020 Mathematics Subject Classification. Primary 11A15; Secondary 11A07, 11 B39. 
Also,

$$
\begin{aligned}
& (-1)^{\left|\left\{1 \leqslant k<n / 2:\{k x\}_{n}>n / 2 \&\{k(1-x)\}_{n}>n / 2\right\}\right|}=\left(\frac{2}{n}\right), \\
& (-1)^{\left|\left\{1 \leqslant k<n / 2:\{k x\}_{n}<n / 2 \&\{k(1-x)\}_{n}<n / 2\right\}\right|}=\left(\frac{2 x(x-1)}{n}\right),
\end{aligned}
$$

and

$$
(-1)^{\left|\left\{1 \leqslant k<n / 2:\{k x\}_{n}>n / 2>\{k(1-x)\}_{n}\right\}\right|}=\left(\frac{2 x}{n}\right) .
$$

Let $p$ be an odd prime, and let $a, b, c \in \mathbb{Z}$ and

$$
S_{p}(a, b, c):=\prod_{\substack{1 \leqslant i<j \leqslant p-1 \\ p \nmid a i^{2}+b i j+c j^{2}}}\left(a i^{2}+b i j+c j^{2}\right) .
$$

Using Theorem 1.1, together with [15, Theorem 1.2], we completely determine $S_{p}(a, b, c) \bmod p$ in terms of Legendre symbols.

Theorem 1.2. Let $p$ be an odd prime, and let $a, b, c \in \mathbb{Z}$ and $\Delta=b^{2}-4 a c$. When $p \nmid a c(a+b+c)$, we have

$$
S_{p}(a, b, c) \equiv \begin{cases}\left(\frac{a(a+b+c)}{p}\right)(\bmod p) & \text { if } p \mid \Delta, \\ -\left(\frac{a c(a+b+c) \Delta}{p}\right)(\bmod p) & \text { if } p \nmid \Delta .\end{cases}
$$

In the case $p \mid a c(a+b+c)$, we have

$$
S_{p}(a, b, c) \equiv \begin{cases}0(\bmod p) & \text { if } p|a, p| b \text { and } p \mid c, \\ -\left(\frac{-a}{p}\right)(\bmod p) & \text { if } p \nmid a, p \mid b \text { and } p \mid c, \\ -\left(\frac{b}{p}\right)(\bmod p) & \text { if } p \mid a, p \nmid b \text { and } p \mid c, \\ -\left(\frac{-c}{p}\right)(\bmod p) & \text { if } p|a, p| b \text { and } p \nmid c, \\ -\left(\frac{c}{p}\right)(\bmod p) & \text { if } p \mid a, p \nmid b c \text { and } p \mid b+c, \\ -\left(\frac{a}{p}\right)(\bmod p) & \text { if } p \nmid a b, p \mid a+b \text { and } p \mid c, \\ -\left(\frac{-a}{p}\right)(\bmod p) & \text { if } p \nmid a c, p \mid a-c \text { and } p \mid a+b+c, \\ \left(\frac{-a c}{p}\right)(\bmod p) & \text { if } p \nmid a c(a-c) \text { and } p \mid a+b+c, \\ \left(\frac{-a(a+b)}{p}\right)(\bmod p) & \text { if } p \nmid a b(a+b) \text { and } p \mid c, \\ \left(\frac{-c(b+c)}{p}\right)(\bmod p) & \text { if } p \mid a \text { and } p \nmid b c(b+c),\end{cases}
$$

We will prove Theorem 1.1 and those parts of Theorem 1.2 not covered by [15, Theorem 1.2] in Section 2.

Let $p$ be an odd prime. For $a, b, c \in \mathbb{Z}$ we introduce

$$
T_{p}(a, b, c):=\prod_{\substack{i, j=1 \\ p \nmid a i^{2}+b i j+c j^{2}}}^{(p-1) / 2}\left(a i^{2}+b i j+c j^{2}\right) .
$$

Our following theorem determines $T_{p}(1,-(a+b),-1)$ modulo $p$ for all $a, b \in \mathbb{Z}$ with $a b \equiv-1(\bmod p)$. 
Theorem 1.3. Let $p$ be any odd prime, and let $a, b \in \mathbb{Z}$ with $a b \equiv-1(\bmod p)$. Set

$$
\{a, b\}_{p}:=\prod_{\substack{i, j=1 \\ i \neq a j, b j(\bmod p)}}^{(p-1) / 2}(i-a j)(i-b j)
$$

which is congruent to $T_{p}(1,-(a+b),-1)$ modulo $p$.

(i) We have

$$
-\{a, b\}_{p} \equiv \begin{cases}\left(\frac{a-b}{p}\right)(\bmod p) & \text { if } p \equiv 1(\bmod 4) \& p \nmid(a-b), \\ \left(\frac{a(a-b)}{p}\right)=\left(\frac{a^{2}+1}{p}\right)(\bmod p) & \text { if } p \equiv 3(\bmod 4) .\end{cases}
$$

(ii) If $a \equiv b(\bmod p)$ and $p \equiv 1(\bmod 8)$, then

$$
\{a, b\}_{p} \equiv(-1)^{(p+7) / 8} \frac{p-1}{2} !(\bmod p) .
$$

If $p \equiv 5(\bmod 8)$ and $a \equiv b \equiv(-1)^{k}((p-1) / 2) !(\bmod p)$ with $k \in\{0,1\}$, then

$$
\{a, b\}_{p} \equiv(-1)^{k+(p-5) / 8}(\bmod p) .
$$

Our proof of Theorem 1.3 will be given in Section 3 .

For any $A \in \mathbb{Z}$, we define the Lucas sequences $\left\{u_{n}(A)\right\}_{n \geqslant 0}$ and $\left\{v_{n}(A)\right\}_{n \geqslant 0}$ by $u_{0}(A)=0, u_{1}(A)=1$, and $u_{n+1}(A)=A u_{n}(A)+u_{n-1}(A)$ for $n=1,2,3, \ldots$, and

$$
v_{0}(A)=2, v_{1}(A)=A, \text { and } v_{n+1}(A)=A v_{n}(A)+v_{n-1}(A) \text { for } n=1,2,3, \ldots
$$

It is well known that

$$
u_{n}(A)=\frac{\alpha^{n}-\beta^{n}}{\alpha-\beta} \text { and } v_{n}(A)=\alpha^{n}+\beta^{n}
$$

for all $n \in \mathbb{N}=\{0,1,2, \ldots\}$, where

$$
\alpha=\frac{A+\sqrt{A^{2}+4}}{2} \text { and } \beta=\frac{A-\sqrt{A^{2}+4}}{2} .
$$

Thus

$$
\left(\frac{A \pm \sqrt{A^{2}+4}}{2}\right)^{n}=\frac{v_{n}(A) \pm u_{n}(A) \sqrt{A^{2}+4}}{2} \text { for all } n \in \mathbb{N} .
$$

Now we state our fourth theorem which determines $T_{p}(1,-A,-1)$ for any odd prime $p$ and integer $A$.

Theorem 1.4. Let $p$ be an odd prime and let $A \in \mathbb{Z}$.

(i) Suppose that $p \mid\left(A^{2}+4\right)$. Then $p \equiv 1(\bmod 4), A / 2 \equiv(-1)^{k}((p-$ $1) / 2)$ ! $(\bmod p)$ for some $k \in\{0,1\}$, and

$$
T_{p}(1,-A,-1) \equiv \begin{cases}(-1)^{(p+7) / 8}((p-1) / 2) !(\bmod p) & \text { if } p \equiv 1(\bmod 8), \\ (-1)^{k+(p-5) / 8}(\bmod p) & \text { if } p \equiv 5(\bmod 8) .\end{cases}
$$

(ii) When $\left(\frac{A^{2}+4}{p}\right)=1$, we have

$$
T_{p}(1,-A,-1) \equiv \begin{cases}-\left(A^{2}+4\right)^{(p-1) / 4}(\bmod p) & \text { if } p \equiv 1(\bmod 4), \\ -\left(A^{2}+4\right)^{(p+1) / 4} u_{(p-1) / 2}(A) / 2(\bmod p) & \text { if } p \equiv 3(\bmod 4) .\end{cases}
$$


(iii) When $\left(\frac{A^{2}+4}{p}\right)=-1$, we have

$T_{p}(1,-A,-1) \equiv \begin{cases}\left(-A^{2}-4\right)^{(p-1) / 4}(\bmod p) & \text { if } p \equiv 1(\bmod 4), \\ \left(-A^{2}-4\right)^{(p+1) / 4} u_{(p+1) / 2}(A) / 2(\bmod p) & \text { if } p \equiv 3(\bmod 4) .\end{cases}$

We will prove Theorem 1.4 in Section 4.

Let $p$ be a prime with $p \equiv 1(\bmod 4)$. Then $\left(\frac{p-1}{2} !\right)^{2} \equiv-1(\bmod p)$ by Wilson's theorem. We may write $p=x^{2}+y^{2}$ with $x, y \in \mathbb{Z}, x \equiv 1(\bmod 4)$ and $y \equiv$ $\frac{p-1}{2} ! x(\bmod p)$. Recall that an integer $a$ not divisible by $p$ is a quartic residue modulo $p$ (i.e., $z^{4} \equiv a(\bmod p)$ for some $\left.z \in \mathbb{Z}\right)$ if and only if $a^{(p-1) / 4} \equiv 1(\bmod p)$. Dirichlet proved that 2 is a quartic residue modulo $p$ if and only if $8 \mid y$ (see, e.g., [6, p. 64, Exer. 28]). On the other hand, we have

$$
\left|\left\{1 \leqslant k<\frac{p}{4}:\left(\frac{k}{p}\right)=1\right\}\right| \equiv 0(\bmod 2) \Longleftrightarrow y \equiv(-1)^{(p-1) / 4}-1(\bmod 8)
$$

as discovered by K. Burde [2] and re-proved by K. S. Williams [16]. In view of Williams and J. D. Currie [17, (1.4)], we have

$$
2^{(p-1) / 4} \equiv(-1)^{\left|\left\{1 \leqslant k<\frac{p}{4}:\left(\frac{k}{p}\right)=-1\right\}\right|} \times \begin{cases}1(\bmod p) & \text { if } p \equiv 1(\bmod 8), \\ \frac{p-1}{2} !(\bmod p) & \text { if } p \equiv 5(\bmod 8) .\end{cases}
$$

By Dirichlet's class number formula (see, e.g., L.E. Dickson [4, p. 101]),

$$
\frac{p-1}{2}-4\left|\left\{1 \leqslant k<\frac{p}{4}:\left(\frac{k}{p}\right)=-1\right\}\right|=h(-4 p),
$$

where $h(d)$ with $d \equiv 0,1(\bmod 4)$ not a square denotes the class number of the quadratic field with discriminant $d$. In 1905, Lerch (see, e.g., [5]) proved that

$$
h(-3 p)=2 \sum_{1 \leqslant k<p / 3}\left(\frac{k}{p}\right) .
$$

By [17, Lemma 14],

$$
(-3)^{(p-1) / 4} \equiv \begin{cases}(-1)^{h(-3 p) / 4}(\bmod p) & \text { if } p \equiv 1(\bmod 12), \\ (-1)^{(h(-3 p)-2) / 4} \frac{p-1}{2} !(\bmod p) & \text { if } p \equiv 5(\bmod 12) .\end{cases}
$$

Thus, if $p \equiv 1(\bmod 12)$ then

$$
(-3)^{(p-1) / 4} \equiv(-1)^{\frac{1}{2} \sum_{k=1}^{(p-1) / 3}\left(\left(\frac{k}{p}\right)-1\right)+\frac{p-1}{6}}=(-1)^{\left|\left\{1 \leqslant k<\frac{p}{3}:\left(\frac{k}{p}\right)=-1\right\}\right|}(\bmod p) ;
$$

similarly, if $p \equiv 5(\bmod 12)$ then

$$
(-3)^{(p-1) / 4} \equiv(-1)^{\left|\left\{1 \leqslant k<\frac{p}{3}:\left(\frac{k}{p}\right)=-1\right\}\right|} \frac{p-1}{2} !(\bmod p) .
$$

From Theorem 1.4, we deduce the following result which will be proved in Section 5 .

Theorem 1.5. Let $p$ be an odd prime. 
(i) We have

$$
T_{p}(1,-1,-1) \equiv \begin{cases}-5^{(p-1) / 4}(\bmod p) & \text { if } p \equiv 1,9(\bmod 20), \\ (-5)^{(p-1) / 4}(\bmod p) & \text { if } p \equiv 13,17(\bmod 20), \\ (-1)^{\lfloor(p-10) / 20\rfloor}(\bmod p) & \text { if } p \equiv 3,7(\bmod 20), \\ (-1)^{\lfloor(p-5) / 10\rfloor}(\bmod p) & \text { if } p \equiv 11,19(\bmod 20) .\end{cases}
$$

(ii) We have

$$
T_{p}(1,-2,-1) \equiv \begin{cases}-2^{(p-1) / 4}(\bmod p) & \text { if } p \equiv 1(\bmod 8), \\ 2^{(p-1) / 4}(\bmod p) & \text { if } p \equiv 5(\bmod 8), \\ (-1)^{(p-3) / 8}(\bmod p) & \text { if } p \equiv 3(\bmod 8), \\ (-1)^{(p-7) / 8}(\bmod p) & \text { if } p \equiv 7(\bmod 8) .\end{cases}
$$

Now we state our sixth theorem.

Theorem 1.6. Let $p>3$ be a prime and let $\delta \in\{ \pm 1\}$. If $p \equiv 1(\bmod 4)$, then

$$
T_{p}(2,5 \delta, 2) \equiv(-1)^{\lfloor(p+11) / 12\rfloor}(\bmod p) .
$$

When $p \equiv 3(\bmod 4)$, we have

$$
T_{p}(2,5 \delta, 2) \equiv\left(\frac{6}{p}\right) \frac{\delta 2^{\delta}}{3^{\delta}}\left(\begin{array}{c}
(p-3) / 2 \\
(p-3) / 4
\end{array}\right)^{-2 \delta}(\bmod p) .
$$

Note that there is no simple closed form for $\left(\begin{array}{l}(p-3) / 2 \\ (p-3) / 4\end{array}\right)$ modulo a prime $p \equiv$ $3(\bmod 4)$. For a prime $p \equiv 1(\bmod 4)$ with $p=x^{2}+y^{2}(x, y \in \mathbb{Z}$ and $x \equiv 1(\bmod 4))$, Gauss showed the congruence $\left(\begin{array}{c}(p-1) / 2 \\ (p-1) / 4\end{array}\right) \equiv 2 x(\bmod p)$, and S. Chowla, B. Dwork and R. J. Evans [3] used Gauss and Jacobi sums to prove further that

$$
\left(\begin{array}{l}
(p-1) / 2 \\
(p-1) / 4
\end{array}\right) \equiv \frac{2^{p-1}+1}{2}\left(2 x-\frac{p}{2 x}\right)\left(\bmod p^{2}\right),
$$

which was first conjectured by F. Beukers. (See also [1, Chapter 9] for further related results.)

Though we have made some numerical tests via a computer, we are unable to find general patterns for $T_{p}(a, b, c)$ modulo $p$, where $p$ is an arbitrary odd prime and $a, b, c$ are arbitrary integers.

Let $p$ be an odd prime. It is known (cf. [15, (1.6) and (1.7)]) that

$$
\prod_{\substack{1 \leqslant i<j \leqslant(p-1) / 2 \\ p \nmid i^{2}+j^{2}}}\left(i^{2}+j^{2}\right) \equiv \begin{cases}(-1)^{\lfloor(p-5) / 8\rfloor}(\bmod p) & \text { if } p \equiv 1(\bmod 4), \\ (-1)^{\lfloor(p+1) / 8\rfloor}(\bmod p) & \text { if } p \equiv 3(\bmod 4) .\end{cases}
$$

From this we immediately get

$$
\prod_{\substack{1 \leqslant i<j \leqslant(p-1) / 2 \\ p \nmid i^{2}+j^{2}}}\left(\frac{i^{2}+j^{2}}{p}\right)= \begin{cases}1 & \text { if } p \equiv 1(\bmod 4), \\ (-1)^{\lfloor(p+1) / 8\rfloor} & \text { if } p \equiv 3(\bmod 4) .\end{cases}
$$

As the product $\prod_{1 \leqslant i<j \leqslant(p-1) / 2}\left(i^{2}-j^{2}\right)$ modulo $p$ was determined via [15, (1.5)], we also know the value of the product

$$
\prod_{1 \leqslant i<j \leqslant(p-1) / 2}\left(\frac{i^{2}-j^{2}}{p}\right)=\prod_{1 \leqslant i<j \leqslant(p-1) / 2}\left(\frac{i-j}{p}\right)\left(\frac{i+j}{p}\right)
$$


Motivated by this, we obtain the following result.

Theorem 1.7. Let $p>3$ be a prime and let $\delta \in\{ \pm 1\}$. Then

$$
\prod_{1 \leqslant i<j \leqslant(p-1) / 2}\left(\frac{j+\delta i}{p}\right)= \begin{cases}(-1)^{\left|\left\{0<k<\frac{p}{4}:\left(\frac{k}{p}\right)=\delta\right\}\right|} & \text { if } p \equiv 1(\bmod 4), \\ (-1)^{(p-3) / 8} & \text { if } p \equiv 3(\bmod 8), \\ (-1)^{(p+1) / 8+(h(-p)+1) / 2} & \text { if } p \equiv 7(\bmod 8) .\end{cases}
$$

We will prove Theorems 1.6 and 1.7 in Section 6, and pose ten conjectures in Section 7.

\section{Proofs of Theorems 1.1 And 1.2}

Proof of Theorem 1.1. For each $k=1, \ldots,(n-1) / 2$, we have

$$
\left\lfloor\frac{k x}{n}\right\rfloor-\left\lfloor\frac{k(x-1)}{n}\right\rfloor= \begin{cases}0 & \text { if }\{k x\}_{n}>k \\ 1 & \text { if }\{k x\}_{n}<k\end{cases}
$$

Thus

$$
\left|\left\{1 \leqslant k<\frac{n}{2}:\{k x\}_{n}>k\right\}\right|=\frac{n-1}{2}-\sum_{k=1}^{(n-1) / 2}\left(\left\lfloor\frac{k x}{n}\right\rfloor-\left\lfloor\frac{k(x-1)}{n}\right\rfloor\right)
$$

and hence

$$
\begin{aligned}
& (-1)^{\left|\left\{1 \leqslant k<n / 2:\{k x\}_{n}>k\right\}\right|}\left(\frac{-1}{n}\right) \\
= & (-1)^{\sum_{k=1}^{(n-1) / 2}\lfloor k x / n\rfloor+\sum_{k=1}^{(n-1) / 2}\lfloor k(x-1) / n\rfloor} \\
= & (-1)^{\left(\sum_{k=1}^{(n-1) / 2}(2 x-1) k-\sum_{k=1}^{(n-1) / 2}\{k x\}_{n}-\sum_{k=1}^{(n-1) / 2}\{k(x-1)\}_{n}\right) / n} \\
= & (-1)^{\left(n^{2}-1\right) / 8}(-1)^{\sum_{k=1}^{(n-1) / 2}\{k x\}_{n}+\sum_{k=1}^{(n-1) / 2}\{k(x-1)\}_{n}} .
\end{aligned}
$$

As $\{k x\}_{n} \equiv 1+n-\{k x\}_{n}(\bmod 2)$ for all $k=1, \ldots,(n-1) / 2$, we have

$$
\begin{aligned}
\sum_{k=1}^{(n-1) / 2}\{k x\}_{n} & \equiv \sum_{\substack{k=1 \\
\{k x\}_{n}<n / 2 \\
(n-1) / 2}}^{(n-1) / 2}\{k x\}_{n}+\sum_{\substack{k=1 \\
\{k x\}_{n}>n / 2}}^{(n-1) / 2}\left(1+\left(n-\{k x\}_{n}\right)\right) \\
& =\sum_{\substack{k=1 \\
\{k x\}_{n}>n / 2}}^{(n-1) / 2} 1+\sum_{r=1}^{(n-1} r \\
& =\left|\left\{1 \leqslant k<\frac{n}{2}:\{k x\}_{n}>\frac{n}{2}\right\}\right|+\frac{n^{2}-1}{8}(\bmod 2) .
\end{aligned}
$$

and hence

$$
(-1)^{\sum_{k=1}^{(n-1) / 2}\{k x\}_{n}}=\left(\frac{x}{n}\right)\left(\frac{2}{n}\right)=\left(\frac{2 x}{n}\right)
$$

with the help of (1.2). Similarly,

$$
(-1)^{\sum_{k=1}^{(n-1) / 2}\{k(x-1)\}_{n}}=\left(\frac{x}{n}\right)\left(\frac{2}{n}\right)=\left(\frac{2(x-1)}{n}\right) .
$$


In view of the above, we obtain

$$
\begin{aligned}
(-1)^{\left|\left\{1 \leqslant k<n / 2:\{k x\}_{n}>k\right\}\right|} & =\left(\frac{-2}{n}\right)(-1)^{\sum_{k=1}^{(n-1) / 2}\{k x\}_{n}}(-1)^{\sum_{k=1}^{(n-1) / 2}\{k(x-1)\}_{n}} \\
& =\left(\frac{-2}{n}\right)\left(\frac{2 x}{n}\right)\left(\frac{2(x-1)}{n}\right)=\left(\frac{2 x(1-x)}{n}\right) .
\end{aligned}
$$

This proves (1.3).

When $1 \leqslant k<n / 2$ and $\{k x\}_{n}<n / 2$, we clearly have

$$
\{k x\}_{n}>k \Longleftrightarrow\{k(1-x)\}_{n}>\frac{n}{2} \text {. }
$$

Thus

$$
\begin{aligned}
& \left|\left\{1 \leqslant k<\frac{n}{2}:\{k x\}_{n}>k\right\}\right|-\left|\left\{1 \leqslant k<\frac{n}{2}:\{k x\}_{n}>\frac{n}{2}\right\}\right| \\
= & \left|\left\{1 \leqslant k<\frac{n}{2}:\{k x\}_{n}<\frac{n}{2}<\{k(1-x)\}_{n}\right\}\right| \\
= & \left|\left\{1 \leqslant k<\frac{n}{2}:\{k(1-x)\}_{n}>\frac{n}{2}\right\}\right| \\
& -\left|\left\{1 \leqslant k<\frac{n}{2}:\{k x\}_{n}>\frac{n}{2} \&\{k(1-x)\}_{n}>\frac{n}{2}\right\}\right|,
\end{aligned}
$$

and hence by (1.2) and (1.3) we get

$$
\begin{aligned}
& (-1)^{\left|\left\{1 \leqslant k<n / 2:\{k x\}_{n}>n / 2 \&\{k(1-x)\}_{n}>n / 2\right\}\right|} \\
= & (-1)^{\left|\left\{1 \leqslant k<n / 2:\{k x\}_{n}>k\right\}\right|}\left(\frac{x}{n}\right)\left(\frac{1-x}{n}\right)=\left(\frac{2}{n}\right) .
\end{aligned}
$$

This proves (1.4).

In view of (1.2) and (1.4), we also have

$$
\begin{aligned}
& (-1)^{\left|\left\{1 \leqslant k<n / 2:\{k x\}_{n}>n / 2>\{k(1-x)\}_{n}\right\}\right|} \\
= & (-1)^{\left|\left\{1 \leqslant k<n / 2:\{k x\}_{n}>n / 2\right\}\right|-||\left\{1 \leqslant k<n / 2:\{k x\}_{n}>n / 2 \&\{k(1-x)\}_{n}>n / 2\right\} \mid} \\
= & \left(\frac{x}{n}\right)\left(\frac{2}{n}\right)=\left(\frac{2 x}{n}\right) .
\end{aligned}
$$

This proves (1.6).

By (1.4) and (1.6), we have

$$
\begin{aligned}
& \left(\frac{2}{n}\right)(-1)^{\left|\left\{1 \leqslant k<n / 2:\{k x\}_{n}<n / 2 \&\{k(1-x)\}_{n}<n / 2\right\}\right|} \\
= & (-1)^{\left|\left\{1 \leqslant k<n / 2:\left(\{k x\}_{n}-n / 2\right)\left(\{k(1-x)\}_{n}-n / 2\right)>0\right\}\right|} \\
= & (-1)^{(n-1) / 2-\mid\left\{1 \leqslant k<n / 2:\{k x\}_{n}>n / 2>\{k(1-x)\}_{n} \text { or }\{k(1-x)\}_{n}>n / 2>\{k x\}_{n}\right\} \mid} \\
= & \left(\frac{-1}{n}\right)\left(\frac{2 x}{n}\right)\left(\frac{2(1-x)}{n}\right)=\left(\frac{x(x-1)}{n}\right) .
\end{aligned}
$$

So (1.5) also holds.

The proof of Theorem 1.1 is now complete.

For any odd prime $p$ and rational $p$-adic integer $x$, we define

$$
N_{p}(x):=\left|\left\{1 \leqslant k<p / 2:\{k x\}_{p}>k\right\}\right| .
$$


Proof of Theorem 1.2. By parts (ii)-(iv) of Sun [15, Theorem 1.2], the desired result holds except for the cases

$$
\begin{aligned}
& \text { I. } p \nmid a c(a-c) \text { and } p \mid a+b+c \text {, } \\
& \text { II. } p \nmid a b(a+b) \text { and } p \mid c, \\
& \text { III. } p \mid a \text { and } p \nmid b c(b+c) \text {. }
\end{aligned}
$$

In case I, by [15, Theorem 1.2(iii)] we have

$$
S_{p}(a, b, c) \equiv(-1)^{N_{p}(a / c)}\left(\frac{2 c(a-c)}{p}\right)(\bmod p) .
$$

As

$$
(-1)^{N_{p}(a / c)}=\left(\frac{2 a(c-a)}{p}\right)
$$

by Theorem 1.1, we obtain

$$
S_{p}(a, b, c) \equiv\left(\frac{2 a(c-a)}{p}\right)\left(\frac{2 c(a-c)}{p}\right)=\left(\frac{-a c}{p}\right)(\bmod p) .
$$

In case II, by [15, Theorem $1.2(\mathrm{iv})]$ we have

$$
S_{p}(a, b, c) \equiv(-1)^{N_{p}(-a / b)}\left(\frac{2}{p}\right)(\bmod p) .
$$

As

$$
(-1)^{N_{p}(-a / b)}=\left(\frac{-2 a(a+b)}{p}\right)
$$

by Theorem 1.1, we get

$$
S_{p}(a, b, c) \equiv\left(\frac{-2 a(a+b)}{p}\right)\left(\frac{2}{p}\right)=\left(\frac{-a(a+b)}{p}\right)(\bmod p) .
$$

In case III, by [15, Theorem 1.2(iv)] we have

$$
S_{p}(a, b, c) \equiv(-1)^{N_{p}(-c / b)}\left(\frac{2}{p}\right)(\bmod p) .
$$

Since $(-1)^{N_{p}(-c / b)}=\left(\frac{-2 c(b+c)}{p}\right)$ by Theorem 1.1, we deduce that

$$
S_{p}(a, b, c) \equiv\left(\frac{-2 c(b+c)}{p}\right)\left(\frac{2}{p}\right)=\left(\frac{-c(b+c)}{p}\right)(\bmod p) .
$$

In view of the above, we have completed the proof of Theorem 1.2.

\section{Proof of Theorem 1.3}

Lemma 3.1. Let $p$ be any odd prime. Then

$$
\left(\frac{p-1}{2} !\right)^{2} \equiv(-1)^{(p+1) / 2}(\bmod p),
$$

and

$$
\prod_{1 \leqslant i<j \leqslant(p-1) / 2}\left(j^{2}-i^{2}\right) \equiv \begin{cases}-((p-1) / 2) !(\bmod p) & \text { if } p \equiv 1(\bmod 4), \\ 1(\bmod p) & \text { if } p \equiv 3(\bmod 4) .\end{cases}
$$

Remark 3.2. (3.1) is an easy consequence of Wilson's theorem. (3.2) is also known, see $[15,(1.5)]$ and its few-line proof there. 
Lemma 3.3. Let $p$ be a prime with $p \equiv 1(\bmod 4)$. Then

$$
\left|\left\{1 \leqslant k \leqslant \frac{p-1}{2}:\left\{k \times \frac{p-1}{2} !\right\}_{p}>\frac{p}{2}\right\}\right|=\frac{p-1}{4} .
$$

Proof. Let $a=((p-1) / 2)$ !. Then $a^{2} \equiv-1(\bmod p)$ by $(3.1)$. For any $k=$ $1, \ldots,(p-1) / 2$, there is a unique integer $k^{*} \in\{1, \ldots,(p-1) / 2\}$ congruent to $a k$ or $-a k$ modulo $p$. Note that $k^{*} \neq k$ since $a \neq \equiv \pm 1(\bmod p)$. If $\{a k\}_{p}>p / 2$ then $\left\{a k^{*}\right\}_{p}=\{a(-a k)\}_{p}=k<p / 2$; if $\{a k\}_{p}<p / 2$ then $\left\{a k^{*}\right\}_{p}=\{a(a k)\}_{p}=p-k>$ $p / 2$. So, exactly one of $\{a k\}_{p}$ and $\left\{a k^{*}\right\}_{p}$ is greater than $p / 2$. Therefore (3.3) holds.

Remark 3.4. In view of Gauss' Lemma, Lemma 3.3 is stronger than the fact that $\left(\frac{((p-1) / 2) !}{p}\right)=\left(\frac{2}{p}\right)$ for any prime $p \equiv 1(\bmod 4)($ cf. [14, Lemma 2.3$\left.]\right)$.

Proof of Theorem 1.3. Observe that

$$
\begin{aligned}
\prod_{\substack{i, j=1 \\
p \nmid i^{2}-j^{2}}}^{(p-1) / 2}\left(i^{2}-j^{2}\right) & =\prod_{1 \leqslant i<j \leqslant(p-1) / 2}\left(i^{2}-j^{2}\right)\left(j^{2}-i^{2}\right) \\
& \left.=(-1)^{(p-1) / 2}\right) \prod_{1 \leqslant i<j \leqslant(p-1) / 2}\left(j^{2}-i^{2}\right)^{2}
\end{aligned}
$$

and hence

$$
\prod_{\substack{i, j=1 \\ p \nmid i^{2}-j^{2}}}^{(p-1) / 2}\left(i^{2}-j^{2}\right) \equiv-\left(\frac{2}{p}\right)(\bmod p)
$$

with the help of Lemma 3.1.

When $a \equiv \pm 1(\bmod p)$, we have $b \equiv \mp 1 \not \equiv a(\bmod p)$ and hence

$$
\{a, b\}_{p} \equiv-\left(\frac{2}{p}\right)= \begin{cases}-\left(\frac{a-b}{p}\right)(\bmod p) & \text { if } p \equiv 1(\bmod 4), \\ -\left(\frac{a^{2}+1}{p}\right)(\bmod p) & \text { if } p \equiv 3(\bmod 4) .\end{cases}
$$

So (1.12) holds in the case $a \equiv \pm 1(\bmod p)$.

Below we assume that $a \not \equiv \pm 1(\bmod p)$. As $a b \equiv-1(\bmod p)$, we have

$$
\begin{aligned}
\{a, b\}_{p} & =\prod_{\substack{i, j=1 \\
i \neq a j, b j(\bmod p) \\
(p-1) / 2}}^{(p-1) / 2}(i-a j) \times \prod_{\substack{i, j=1 \\
j \neq a i, b i \\
(\bmod p)}}^{(p-1) / 2}(j-b i) \\
& \equiv \prod_{\substack{i, j=1 \\
p \nmid(i-a j)(j+a i)}}^{(p-1) / 2}(i-a j) \times \prod_{\substack{i, j=1 \\
p \nmid(i+a j)(j-a i)}}^{(i+a j} \frac{i \bmod p) .}{a}(\log )
\end{aligned}
$$

(i) If $p \equiv 3(\bmod 4)$, then $\left(\frac{-1}{p}\right)=-1$ and hence $a \not \equiv b(\bmod p)$. Now we prove (1.12) under the assumption $a \not \equiv b(\bmod p)$. Note that $a^{2} \not \equiv \pm 1(\bmod p)$. 
For $1 \leqslant i, j \leqslant(p-1) / 2$, we cannot have $i^{2}-a^{2} j^{2} \equiv j^{2}-a^{2} i^{2} \equiv 0(\bmod p)$. Thus

$$
\begin{aligned}
& \left|\left\{(i, j): 1 \leqslant i, j \leqslant \frac{p-1}{2} \& p \nmid(i+a j)(j-a i)\right\}\right| \\
= & \left(\frac{p-1}{2}\right)^{2}-\left|\left\{(i, j): 1 \leqslant i, j \leqslant \frac{p-1}{2} \& p \mid i+a j\right\}\right| \\
& -\left|\left\{(i, j): 1 \leqslant i, j \leqslant \frac{p-1}{2} \& p \mid j-a i\right\}\right| \\
= & \left(\frac{p-1}{2}\right)^{2}-\left|\left\{1 \leqslant j \leqslant \frac{p-1}{2}:\{a j\}_{p}>\frac{p}{2}\right\}\right| \\
& -\left|\left\{1 \leqslant i \leqslant \frac{p-1}{2}:\{a i\}_{p}<\frac{p}{2}\right\}\right| \\
= & (p-1) \frac{p-1}{4}-\frac{p-1}{2}=\frac{p-1}{2} \cdot \frac{p+1}{2}-(p-1)
\end{aligned}
$$

and hence

$a^{|\{(i, j): 1 \leqslant i, j \leqslant(p-1) / 2 \& p \nmid(i+a j)(j-a i)\}|} \equiv\left(a^{(p-1) / 2}\right)^{(p+1) / 2} \equiv\left(\frac{a}{p}\right)^{(p+1) / 2}(\bmod p)$.

Combining this with (3.5), we obtain

$$
\begin{aligned}
& \left(\frac{a}{p}\right)^{(p+1) / 2}\{a, b\}_{p} \\
\equiv & \prod_{\substack{i, j=1 \\
p \nmid\left(i^{2}-a^{2} j^{2}\right)\left(j^{2}-a^{2} i^{2}\right)}}^{(p-1) / 2}\left(i^{2}-a^{2} j^{2}\right) \times \prod_{\substack{i, j=1 \\
i \equiv a j(\bmod p)}}^{(p-1) / 2}(i+a j) \times \prod_{\substack{i, j=1 \\
i \equiv-a j(\bmod p)}}^{(p-1) / 2}(i-a j) \\
& \times \prod_{\substack{i, j=1 \\
j-a i \equiv-a(i+b j) \equiv 0(\bmod p)}}^{(p-1) / 2}(i+a j) \\
\equiv & \prod_{\substack{i, j=1 \\
p \nmid i^{2}-a^{2} j^{2}}}^{(p-1) / 2}\left(i^{2}-a^{2} j^{2}\right) / \prod_{\substack{i, j=1 \\
p \mid j^{2}-a^{2} i^{2}}}^{(p-1) / 2}\left(i^{2}-a^{2} j^{2}\right) \\
& \times(-1)^{\left|\left\{1 \leqslant j \leqslant(p-1) / 2:\{a j\}_{p}>p / 2\right\}\right|} \prod_{j=1}^{(p-1) / 2}(2 a j) \\
& \times(-1)^{\left|\left\{1 \leqslant j \leqslant(p-1) / 2:\{b j\}_{p}>p / 2\right\}\right|} \prod_{j=1}^{(p-1) / 2}(b j+a j)(\bmod p)
\end{aligned}
$$


Thus, by using (3.4) and Gauss' Lemma, we see that

$$
\begin{aligned}
\left(\frac{a}{p}\right)^{(p+1) / 2}\{a, b\}_{p} \equiv & \prod_{\substack{i, k=1 \\
p \nmid i^{2}-k^{2}}}^{(p-1) / 2}\left(i^{2}-k^{2}\right) / \prod_{i=1}^{(p-1) / 2}\left(i^{2}-a^{2}\left(a^{2} i^{2}\right)\right) \\
& \times\left(\frac{a b}{p}\right)(2 a(a+b))^{(p-1) / 2}\left(\frac{p-1}{2} !\right)^{2} \\
\equiv & -\left(\frac{2}{p}\right)\left(\frac{-1}{p}\right)\left(\frac{2\left(a^{2}-1\right)\left(1-a^{4}\right)}{p}\right) \\
= & -\left(\frac{a^{2}+1}{p}\right)=-\left(\frac{a^{2}-a b}{p}\right)(\bmod p) .
\end{aligned}
$$

This proves (1.12).

(ii) Now we come to prove part (ii) of Theorem 1.3. Assume that $a \equiv b(\bmod p)$. Then $a^{2} \equiv a b \equiv-1(\bmod p)$ and hence $p \equiv 1(\bmod 4)$. As $j \pm a i \equiv \pm a(i \mp$ aj) $(\bmod p)$, by $(3.5)$ we have

$$
\begin{aligned}
\{a, b\}_{p} \equiv & a^{-|\{(i, j): 1 \leqslant i, j \leqslant(p-1) / 2 \& p \nmid i+a j\}|} \prod_{\substack{i, j=1 \\
p \nmid i-a j}}^{(p-1) / 2}(i-a j) \times \prod_{\substack{i, j=1 \\
p \nmid i+a j}}^{(p-1) / 2}(i+a j) \\
\equiv & \left.a^{-(p-1)^{2} / 4+\mid\{(i, j): 1 \leqslant i, j \leqslant(p-1) / 2} \& p \mid i+a j\right\} \mid \\
& \times \prod_{\substack{i, j=1 \\
p \nmid i^{2}-(a j)^{2}}}^{(p-1) / 2}\left(i^{2}-(a j)^{2}\right) \\
\equiv & \prod^{|\{(i, j): 1 \leqslant i, j \leqslant(p-1) / 2 \& p \mid i+a j\}|} \prod_{\substack{i, k=1 \\
p \mid i-a j}}^{(p-1) / 2}(i+a j) \times \prod_{\substack{i, j=1 \\
p \nmid i+a j}}^{(p-1) / 2}(i-a j) \\
& \times(-1)^{\left|\left\{1 \leqslant j \leqslant(p-1) / 2:\{a j\}_{p}>p / 2\right\}\right|} \prod_{j=1}^{(p-1) / 2}(2 a j) .(\bmod p)
\end{aligned}
$$

Applying (3.4) and Gauss' Lemma, from the above we get

$$
\begin{aligned}
\{a, b\}_{p} & \equiv a^{\left|\left\{1 \leqslant j \leqslant(p-1) / 2:\{a j\}_{p}>p / 2\right\}\right|} \times\left(-\left(\frac{2}{p}\right)\right)\left(\frac{a}{p}\right)(2 a)^{(p-1) / 2} \frac{p-1}{2} ! \\
& \equiv-\frac{p-1}{2} ! \times a^{\left|\left\{1 \leqslant j \leqslant(p-1) / 2:\{a j\}_{p}>p / 2\right\}\right|}(\bmod p) .
\end{aligned}
$$

As $\left.a^{2} \equiv-1 \equiv((p-1) / 2) !\right)^{2}$, we have $a \equiv(-1)^{k}((p-1) / 2) !(\bmod p)$ for some $k \in\{0,1\}$. In view of Lemma 3.3,

$$
\begin{aligned}
& \left|\left\{1 \leqslant j \leqslant \frac{p-1}{2}:\left\{-\frac{p-1}{2} ! j\right\}_{p}>\frac{p}{2}\right\}\right| \\
= & \left|\left\{1 \leqslant j \leqslant \frac{p-1}{2}:\left\{\frac{p-1}{2} ! j\right\}_{p}<\frac{p}{2}\right\}\right|=\frac{p-1}{4} .
\end{aligned}
$$


Hence

$$
\begin{aligned}
& a^{\left|\left\{1 \leqslant j \leqslant(p-1) / 2:\{a j\}_{p}>p / 2\right\}\right|} \\
= & a^{(p-1) / 4}=(-1)^{k(p-1) / 4}\left(\frac{p-1}{2} !\right)^{(p-1) / 4} \\
\equiv & \begin{cases}\left(\frac{p-1}{2} !\right)^{2(p-1) / 8} \equiv(-1)^{(p-1) / 8}(\bmod p) & \text { if } p \equiv 1(\bmod 8), \\
(-1)^{k} \frac{p-1}{2} !\left(\frac{p-1}{2} !\right)^{2(p-5) / 8} \equiv(-1)^{k+(p-5) / 8} \frac{p-1}{2} !(\bmod p) & \text { if } p \equiv 5(\bmod 8) .\end{cases}
\end{aligned}
$$

Combining this with (3.6) we immediately obtain the desired results in Theorem $1.3($ ii).

In view of the above, we have completed the proof of Theorem 1.3.

\section{Proof of Theorem 1.4}

Proof of Theorem 1.4(i). As $A^{2} \equiv-4(\bmod p)$, we have $\left(\frac{-1}{p}\right)=1$ and hence $p \equiv$ $1(\bmod 4)$. Since $((p-1) / 2)^{2} \equiv-1 \equiv(A / 2)^{2}(\bmod p)$, for some $k \in\{0,1\}$ we have $A / 2 \equiv(-1)^{k}((p-1) / 2) !(\bmod p)$. Choose $a, b \in \mathbb{Z}$ with $a \equiv b \equiv A / 2(\bmod p)$. Note that $\{a, b\}_{p} \equiv T_{p}(1,-A,-1)(\bmod p)$. Applying Theorem 1.3(ii) we immediately get the desired (1.14).

For any odd prime $p$ and integer $A$ with $\Delta=A^{2}+4 \not \equiv 0(\bmod p)$, it is known (cf. [13, Lemma 2.3]) that

$$
u_{\left(p-\left(\frac{\Delta}{p}\right) / 2\right.}(A) v_{\left(p-\left(\frac{\Delta}{p}\right)\right) / 2}(A)=u_{p-\left(\frac{\Delta}{p}\right)}(A) \equiv 0(\bmod p) .
$$

Lemma 4.1. Let $A \in \mathbb{Z}$ and let $p$ be an odd prime not dividing $\Delta=A^{2}+4$. Then

$$
p \mid v_{\left(p-\left(\frac{\Delta}{p}\right)\right) / 2}(A) \Longleftrightarrow\left(\frac{-1}{p}\right)=-1 .
$$

Remark 4.2. This is a known result, see, e.g., [10, Chapter 2, (IV.23)].

Proof of Theorem 1.4(ii). Let $\Delta=A^{2}+4$. As $\left(\frac{\Delta}{p}\right)=1$, we have $d^{2} \equiv \Delta$ for some $d \in \mathbb{Z}$ with $p \nmid d$. Choose integers $a$ and $b$ such that

$$
a \equiv \frac{A+d}{2}(\bmod p) \text { and } b \equiv \frac{A-d}{2}(\bmod p) .
$$

Then $a+b \equiv A(\bmod p)$ and $a b \equiv\left(A^{2}-d^{2}\right) / 4 \equiv-1(\bmod p)$. Thus, for any $i, j \in \mathbb{Z}$ we have

$$
i^{2}-A i j-j^{2} \equiv(i-a j)(i-b j)(\bmod p) .
$$

If $p \equiv 1(\bmod 4)$, then

$$
\left(A^{2}+4\right)^{(p-1) / 4} \equiv d^{(p-1) / 2} \equiv(a-b)^{(p-1) / 2} \equiv\left(\frac{a-b}{p}\right)(\bmod p) .
$$

If $p \equiv 3(\bmod 4)$, then

$$
\left(\frac{2 d(A+d)}{p}\right)=\left(\frac{a d}{p}\right)=\left(\frac{a(a-b)}{p}\right) .
$$


For any positive integer $n$, clearly

$$
\begin{aligned}
u_{n}(A) & =\frac{1}{\sqrt{\Delta}}\left(\left(\frac{A+\sqrt{\Delta}}{2}\right)^{n}-\left(\frac{A-\sqrt{\Delta}}{2}\right)^{n}\right) \\
& =\frac{1}{2^{n-1}} \sum_{k=0}^{\lfloor(n-1) / 2\rfloor}\left(\begin{array}{c}
n \\
2 k+1
\end{array}\right) A^{n-1-2 k} \Delta^{k} \\
& \equiv \frac{1}{2^{n-1}} \sum_{k=0}^{\lfloor(n-1) / 2\rfloor}\left(\begin{array}{c}
n \\
2 k+1
\end{array}\right) A^{n-1-2 k} d^{2 k} \\
& =\frac{1}{d}\left(\left(\frac{A+d}{2}\right)^{n}-\left(\frac{A-d}{2}\right)^{n}\right)(\bmod p)
\end{aligned}
$$

and

$$
\begin{aligned}
v_{n}(A) & =\left(\frac{A+\sqrt{\Delta}}{2}\right)^{n}+\left(\frac{A-\sqrt{\Delta}}{2}\right)^{n}=\frac{1}{2^{n-1}} \sum_{k=0}^{\lfloor n / 2\rfloor}\left(\begin{array}{c}
n \\
2 k
\end{array}\right) A^{n-2 k} \Delta^{k} \\
& \equiv \frac{1}{2^{n-1}} \sum_{k=0}^{\lfloor n / 2\rfloor}\left(\begin{array}{c}
n \\
2 k
\end{array}\right) A^{n-2 k} d^{2 k}=\left(\frac{A+d}{2}\right)^{n}+\left(\frac{A-d}{2}\right)^{n}(\bmod p) .
\end{aligned}
$$

In the case $p \equiv 3(\bmod 4)$, we have $p \mid v_{(p-1) / 2}(A)$ by Lemma 4.1 , and hence

$$
\begin{aligned}
\left(\frac{2 \delta(A+d)}{p}\right) & \equiv d^{(p-1) / 2}\left(\frac{A+d}{2}\right)^{(p-1) / 2} \equiv d^{(p-1) / 2} \frac{v_{(p-1) / 2}(A)+d u_{(p-1) / 2}(A)}{2} \\
& \equiv\left(d^{2}\right)^{(p+1) / 4} \frac{u_{(p-1) / 2}(A)}{2} \equiv \Delta^{(p+1) / 4} \frac{u_{(p-1) / 2}(A)}{2}(\bmod p) .
\end{aligned}
$$

In view of the above, we obtain (1.15) from Theorem 1.3(i).

Lemma 4.3. Let $p$ be an odd prime, and let $A \in \mathbb{Z}$ with $\Delta=A^{2}+4 \not \equiv 0(\bmod p)$. If $p \equiv 1(\bmod 4)$, then

$$
u_{\left(p+\left(\frac{\Delta}{p}\right)\right) / 2}(A) \equiv \pm \Delta^{(p-1) / 4}(\bmod p) .
$$

If $p \equiv 3(\bmod 4)$, then

$$
u_{\left(p-\left(\frac{\Delta}{p}\right)\right) / 2}(A) \equiv \pm 2 \Delta^{(p-3) / 4}(\bmod p) .
$$

Remark 4.4. This is a known result, see, e.g., [11, Theorem 4.1].

Proof of Theorem 1.4(iii). Let

$$
\Delta=A^{2}+4, \alpha=\frac{A+\sqrt{\Delta}}{2} \text { and } \beta=\frac{A-\sqrt{\Delta}}{2} .
$$


As $x^{2}-A x-1=(x-\alpha)(x-\beta)$, both $\alpha$ and $\beta$ are algebraic integers. Observe that

$$
\begin{aligned}
& \prod_{i, j=1}^{(p-1) / 2}\left(i^{2}-A i j-j^{2}\right) \\
= & \prod_{i, j=1}^{(p-1) / 2}(i-\alpha j)(i-\beta j)=\prod_{i, j=1}^{(p-1) / 2}(i-\alpha j) \times \prod_{i, j=1}^{(p-1) / 2}(j-\beta i) \\
= & \prod_{i, j=1}^{(p-1) / 2}(i-\alpha j) \times \prod_{i, j=1}^{(p-1) / 2} \frac{\alpha j+i}{\alpha}=\alpha^{-((p-1) / 2)^{2}} \prod_{i, j=1}^{(p-1) / 2}\left(i^{2}-\alpha^{2} j^{2}\right) \\
= & \alpha^{-((p-1) / 2)^{2}} \prod_{i, j=1}^{(p-1) / 2}\left(-j^{2}\right)\left(\alpha^{2}-\frac{i^{2}}{j^{2}}\right) \\
= & (-\alpha)^{-((p-1) / 2)^{2}}\left(\frac{p-1}{2} !\right)^{2 \frac{p-1}{2}} \prod_{j=1}^{(p-1) / 2} \prod_{i=1}^{(p-1) / 2}\left(\alpha^{2}-\frac{i^{2}}{j^{2}}\right)
\end{aligned}
$$

and hence

$$
\prod_{i, j=1}^{(p-1) / 2}\left(i^{2}-A i j-j^{2}\right) \equiv \beta^{((p-1) / 2)^{2}} \prod_{k=1}^{(p-1) / 2}\left(\alpha^{2}-k^{2}\right)^{(p-1) / 2}(\bmod p)
$$

(in the ring of all algebraic integers) since for any $j, k=1, \ldots,(p-1) / 2$ there is a unique $i \in\{1, \ldots,(p-1) / 2\}$ congruent to $j k$ or $-j k$ modulo $p$. Note that

$$
\prod_{x=1}^{(p-1) / 2}\left(x-k^{2}\right) \equiv x^{(p-1) / 2}-1(\bmod p)
$$

Thus

$$
\begin{aligned}
\prod_{i, j=1}^{(p-1) / 2}\left(i^{2}-A i j-j^{2}\right) & \equiv \beta^{((p-1) / 2)^{2}}\left(\left(\alpha^{2}\right)^{(p-1) / 2}-1\right)^{(p-1) / 2} \\
& =\beta^{((p-1) / 2)^{2}}\left(\alpha^{p-1}-1\right)^{(p-1) / 2} \\
& =\left((-\alpha)^{(p-1) / 2}-\beta^{(p-1) / 2}\right)^{(p-1) / 2}(\bmod p) .
\end{aligned}
$$

Case 1. $p \equiv 1(\bmod 4)$.

In this case,

$$
\begin{aligned}
\prod_{i, j=1}^{(p-1) / 2}\left(i^{2}-A i j-j^{2}\right) & \equiv\left(\frac{\alpha^{(p-1) / 2}-\beta^{(p-1) / 2}}{\alpha-\beta}\right)^{(p-1) / 2}(\alpha-\beta)^{(p-1) / 2} \\
& =u_{(p-1) / 2}(A)^{(p-1) / 2} \Delta^{(p-1) / 4}(\bmod p) .
\end{aligned}
$$

As $\left(\frac{\Delta}{p}\right)=-1$, by Lemma 4.3 we have $u_{(p-1) / 2}(A) \equiv \pm \Delta^{(p-1) / 4}(\bmod p)$ and hence

$$
u_{(p-1) / 2}(A)^{(p-1) / 2} \equiv \Delta^{\frac{p-1}{2} \cdot \frac{p-1}{4}} \equiv(-1)^{(p-1) / 4}(\bmod p) .
$$

Therefore

$$
\prod_{i, j=1}^{(p-1) / 2}\left(i^{2}-A i j-j^{2}\right) \equiv(-\Delta)^{(p-1) / 4}(\bmod p) .
$$


Case 2. $p \equiv 3(\bmod 4)$.

In this case,

$$
\begin{aligned}
\prod_{i, j=1}^{(p-1) / 2}\left(i^{2}-A i j-j^{2}\right) & \equiv-\left(\alpha^{(p-1) / 2}+\beta^{(p-1) / 2}\right)^{(p-1) / 2} \\
& =-v_{(p-1) / 2}(A)^{(p-1) / 2}(\bmod p) .
\end{aligned}
$$

It is easy to see that $2 v_{n-1}(A)=\Delta u_{n}(A)-A v_{n}(A)$ for all $n=1,2,3, \ldots$ Hence

$$
2 v_{(p-1) / 2}(A)=\Delta u_{(p+1) / 2}(A)-A v_{(p+1) / 2}(A) \equiv \Delta u_{(p+1) / 2}(A)(\bmod p)
$$

since $v_{(p+1) / 2}(A)=v_{\left(p-\left(\frac{\Delta}{p}\right)\right) / 2}(A) \equiv 0(\bmod p)$ by Lemma 4.1. Thus

$$
\begin{aligned}
\prod_{i, j=1}^{(p-1) / 2}\left(i^{2}-A i j-j^{2}\right) & \equiv-\left(\frac{\Delta}{2} u_{(p+1) / 2}(A)\right)^{(p-1) / 2} \\
& \equiv \frac{u_{(p+1) / 2}(A)}{2}\left(\frac{u_{(p+1) / 2}(A)}{2}\right)^{(p-3) / 2}(\bmod p) .
\end{aligned}
$$

As $\left(\frac{\Delta}{p}\right)=-1$, by Lemma 4.3 we have $u_{(p+1) / 2}(A) \equiv \pm 2 \Delta^{(p-3) / 4}(\bmod p)$, and hence

$$
\begin{aligned}
\prod_{i, j=1}^{(p-1) / 2}\left(i^{2}-A i j-j^{2}\right) & \equiv \frac{u_{(p+1) / 2}(A)}{2} \Delta^{\frac{p-3}{4} \cdot \frac{p-3}{2}}=\frac{u_{(p+1) / 2}(A)}{2} \Delta^{\frac{p+1}{2} \cdot \frac{p+1}{4}-(p-1)} \\
& \equiv \frac{u_{(p+1) / 2}(A)}{2}(-\Delta)^{(p+1) / 4}(\bmod p) .
\end{aligned}
$$

In view of the above, we have completed the proof of Theorem 1.4(iii).

\section{Proof of Theorem 1.5}

Proof Theorem 1.5. Note that $\left\{u_{n}(1)\right\}_{n \geqslant 0}$ is just the Fibonacci sequence $\left\{F_{n}\right\}_{n \geqslant 0}$. By $\left[12\right.$, Corollary $2($ iii) $)$, if $p \equiv 3(\bmod 4)$ and $\left(\frac{5}{p}\right)=1$, then

$$
u_{(p-1) / 2}(1)=F_{(p-1) / 2} \equiv-2(-1)^{\lfloor(p-5) / 10\rfloor} 5^{(p-3) / 4}(\bmod p)
$$

and hence

$$
-5^{(p+1) / 4} \frac{u_{(p-1) / 2}(1)}{2} \equiv(-1)^{\lfloor(p-5) / 10\rfloor} 5^{(p+1) / 4+(p-3) / 4} \equiv(-1)^{\lfloor(p-5) / 10\rfloor}(\bmod p) .
$$

Similarly, if $p \equiv 3(\bmod 4)$ and $\left(\frac{5}{p}\right)=-1$, then

$$
u_{(p+1) / 2}(1)=F_{(p+1) / 2} \equiv 2(-1)^{\lfloor(p-5) / 10\rfloor} 5^{(p-3) / 4}(\bmod p)
$$

and hence

$$
5^{(p+1) / 4} \frac{u_{(p+1) / 2}(1)}{2} \equiv(-1)^{\lfloor(p-5) / 10\rfloor} 5^{(p+1) / 4+(p-3) / 4} \equiv(-1)^{\lfloor(p+5) / 10\rfloor}(\bmod p) .
$$

Therefore Theorem 1.4 with $A=1$ yields (1.17).

When $p \equiv 1(\bmod 4)$, we obviously have

$$
8^{(p-1) / 4}=2^{(p-1) / 2+(p-1) / 4} \equiv\left(\frac{2}{p}\right) 2^{(p-1) / 4}=(-2)^{(p-1) / 4}(\bmod p) .
$$


If $p \equiv 7(\bmod 8)$, then

$$
u_{(p-1) / 2}(2) \equiv(-1)^{(p+1) / 8} 2^{(p-3) / 4}(\bmod p)
$$

by $[11,(1.7)]$, and hence

$$
-8^{(p+1) / 4} \frac{u_{(p-1) / 2}(2)}{2} \equiv-2^{(3 p+3) / 4}(-1)^{(p+1) / 8} 2^{(p-7) / 4} \equiv(-1)^{(p-7) / 8}(\bmod p) .
$$

Similarly, if $p \equiv 3(\bmod 8)$, then

$$
u_{(p+1) / 2}(2) \equiv(-1)^{(p+5) / 8} 2^{(p-3) / 4}(\bmod p)
$$

by $[11,(1.7)]$, and hence

$$
(-8)^{(p+1) / 4} \frac{u_{(p+1) / 2}(2)}{2} \equiv-2^{(3 p+3) / 4}(-1)^{(p+5) / 8} 2^{(p-7) / 4} \equiv(-1)^{(p-3) / 8}(\bmod p) .
$$

So Theorem 1.4 with $A=2$ yields (1.18). This ends the proof.

\section{Proofs of Theorems 1.6 and 1.7}

For $n=1,2,3, \ldots$, we adopt the notation

$$
n ! !:=\prod_{k=0}^{\lfloor(n-1) / 2\rfloor}(n-2 k) .
$$

Lemma 6.1. Let $p>3$ be a prime. Then

$$
\frac{p-1}{2} ! ! \prod_{\substack{i, j=1 \\ p \nmid 2 i+j}}^{(p-1) / 2}(2 i+j) \equiv\left(\frac{-2}{p}\right) \frac{p-3}{2} ! ! \prod_{\substack{i, j=1 \\ p \nmid 2 i-j}}^{(p-1) / 2}(2 i-j) \equiv \pm 1(\bmod p) \text {. }
$$

Proof. Set

$$
A_{p}:=\frac{p-1}{2} ! ! \prod_{\substack{i, j=1 \\ p \nmid 2 i+j}}^{(p-1) / 2}(2 i+j) \text { and } B_{p}:=\frac{p-3}{2} ! ! \prod_{\substack{i, j=1 \\ p \nmid 2 i-j}}^{(p-1) / 2}(2 i-j) .
$$

Then

$$
\begin{aligned}
\frac{A_{p} B_{p}}{((p-1) / 2) !} & \equiv \prod_{\substack{i, j=1 \\
p \nmid 2 i+j}}^{(p-1) / 2}(2 i+j) \times \prod_{\substack{i, j=1 \\
p+2 i+p-j}}^{(p-1) / 2}(2 i+p-j)=\prod_{i=1}^{(p-1) / 2}\left(\frac{1}{2 i} \prod_{\substack{j=0 \\
p \nmid 2 i+j}}^{p-1}(2 i+j)\right) \\
& \equiv \prod_{i=1}^{(p-1) / 2} \frac{(p-1) !}{2 i} \equiv \frac{\left(\frac{-2}{p}\right)}{((p-1) / 2) !}(\bmod p)
\end{aligned}
$$

and hence

$$
A_{p} B_{p} \equiv\left(\frac{-2}{p}\right)(\bmod p)
$$


On the other hand,

$$
\begin{aligned}
\frac{B_{p}}{((p-3) / 2) ! !}= & \prod_{\substack{i, j=1 \\
p \nmid 2\left(\frac{p+1}{2}-i\right)-j}}^{(p-1) / 2}\left(2\left(\frac{p+1}{2}-i\right)-j\right) \\
\equiv & \prod_{\substack{i, j=1 \\
p \nmid 2 i+j-1}}^{(p-1) / 2}(1-j-2 i)=\prod_{i=1}^{(p-1) / 2} \frac{-2 i}{(-(p-1) / 2-2 i)^{*}} \prod_{\substack{j=1 \\
p \nmid j+2 i}}^{(p-1) / 2}(-j-2 i) \\
\equiv & \frac{(-2)^{(p-1) / 2}((p-1) / 2) !}{\prod_{\substack{1 \leqslant i<p / 2 \\
4 i \neq p+1}}((p+1) / 2-2 i)} \prod_{\substack{i, j=1 \\
p \nmid 2 i+j}}^{(p-1) / 2}(2 i+j) \\
& \times(-1)^{\sum_{i=1}^{(p-1) / 2}((p-1) / 2-|\{1 \leqslant j \leqslant(p-1) / 2: p \mid 2 i+j\}|)}(\bmod p),
\end{aligned}
$$

where $k^{*}$ is 1 or $k$ according as $p \mid k$ or not. Note that

$$
\prod_{1 \leqslant i<p / 4}\left(\frac{p+1}{2}-2 i\right)=\frac{p-3}{2} ! ! .
$$

Therefore

$$
\begin{aligned}
\left(\frac{-2}{p}\right) \frac{B_{p}}{A_{p}} \equiv & \frac{((p-1) / 2) !}{((p-1) / 2) ! !} \times \prod_{(p+1) / 4<i<p / 2} \frac{1}{((p+1) / 2-2 i)} \\
& \times(-1)^{((p-1) / 2)^{2}-|\{1 \leqslant i \leqslant(p-1) / 2: 2 i>p / 2\}|} \\
\equiv & \frac{((p-3) / 2) ! !(-1)^{((p-1) / 2)^{2}-((p-1) / 2-\lfloor(p-1) / 4\rfloor)}}{(-1)^{\lfloor(p-1) / 4\rfloor} \prod_{(p+1) / 4<i<p / 2}(2 i-(p+1) / 2)}=1(\bmod p),
\end{aligned}
$$

and hence

$$
A_{p} \equiv\left(\frac{-2}{p}\right) B_{p}(\bmod p)
$$

which gives the first congruence in (6.1).

Combining $(6.2)$ and $(6.3)$, we see that $A_{p}^{2} \equiv B_{p}^{2} \equiv 1(\bmod p)$. So $(6.1)$ does hold. This ends the proof.

Proof of Theorem 1.6. As $2 i^{2}+\delta 5 i j+2 j^{2}=(i+\delta 2 j)(2 i+\delta j)$, we have

$$
\begin{aligned}
\prod_{\substack{i, j=1 \\
p \nmid 2 i^{2}+\delta 5 i j+2 j^{2}}}^{(p-1) / 2}\left(2 i^{2}+\delta 5 i j+2 j^{2}\right) & =\prod_{\substack{i, j=1 \\
p \nmid(i+\delta 2 j)(2 i+\delta j)}}^{(p-1) / 2}(i+\delta 2 j) \times \prod_{\substack{i, j=1 \\
p \nmid(i+\delta 2 j)(2 i+\delta j)}}^{(p-1) / 2}(2 i+\delta j) \\
& =\prod_{\substack{i, j=1 \\
p \nmid \nmid i+\delta 2 j)(2 i+\delta j)}}^{(p-1) / 2}(i+\delta 2 j) \times \prod_{\substack{i, j=1 \\
p \nmid(i+\delta 2 j)(2 i+\delta j)}}^{(p-1) / 2}(2 j+\delta i) \\
& =\prod_{\substack{i, j=1 \\
p \nmid i+\delta 2 j}}^{(p-1) / 2}(i+\delta 2 j)^{2} \times \prod_{\substack{i, j=1 \\
p \nmid j+\delta 2 i}}^{(p-1) / 2} \frac{1}{\delta(i+\delta 2 j)^{2}} .
\end{aligned}
$$


(Note that $i+\delta 2 j \equiv j+\delta 2 i \equiv 0(\bmod p)$ for no $i, j=1, \ldots,(p-1) / 2$.) Thus, applying Lemma 6.1 and (3.1) we get

$$
\begin{aligned}
& \prod_{\substack{i, j=1 \\
p \nmid 2 i^{2}+\delta 5 i j+2 j^{2}}}^{(p-1) / 2}\left(2 i^{2}+\delta 5 i j+2 j^{2}\right) \\
& \equiv \frac{\delta^{\mid(i, j): 1 \leqslant i, j \leqslant(p-1) / 2 \& p \nmid i+\delta 2 j\} \mid}}{((p-2+\delta) / 2) ! !^{2}} \times \prod_{\substack{i=1 \\
\{\delta 2 i\}_{p}>p / 2}}^{(p-1) / 2} \frac{1}{\delta(i+\delta 2(p-2 \delta i))^{2}} \\
& \equiv \frac{\delta^{((p-1) / 2)^{2}-\left|\left\{1 \leqslant j \leqslant(p-1) / 2:\{\delta 2 j\}_{p}>p / 2\right\}\right|}}{((p-2+\delta) / 2) !^{2}} \times \prod_{\substack{i=1 \\
\{\delta 2 i\}_{p}>p / 2}}^{(p-1) / 2} \frac{1}{\delta(i-4 i)^{2}} \\
& \equiv \frac{\delta^{(p-1)^{2} / 4}}{((p-2+\delta) / 2) ! !^{2}} \times \prod_{i=1}^{(p-1) / 2}(3 i)^{-1-\delta} \times \prod_{i=1}^{\lfloor p / 4\rfloor}(3 i)^{2 \delta} \\
& \equiv \frac{\delta^{(p-1)^{2} / 4}}{((p-2+\delta) / 2) ! !^{2}} \times \frac{3^{2 \delta\lfloor p / 4\rfloor}\lfloor p / 4\rfloor !^{2 \delta}}{((p-1) / 2) !^{1+\delta}} \\
& \equiv \delta^{(p-1)^{2} / 4}(-1)^{\frac{p+1}{2} \cdot \frac{1+\delta}{2}} 3^{2 \delta\lfloor p / 4\rfloor}\left(\frac{\lfloor p / 4\rfloor !^{\delta}}{((p-2+\delta) / 2) ! !}\right)^{2} \\
& =(-1)^{(p+\delta) / 2} 3^{2 \delta\lfloor p / 4\rfloor}\left(\frac{\lfloor p / 4\rfloor !^{\delta}}{((p-2+\delta) / 2) ! !}\right)^{2}(\bmod p) .
\end{aligned}
$$

Case 1. $p \equiv 1(\bmod 4)$.

In this case,

$$
\left(\frac{\lfloor p / 4\rfloor !^{\delta}}{((p-2+\delta) / 2) ! !}\right)^{2}= \begin{cases}1 / 2^{(p-1) / 2} & \text { if } \delta=1, \\ 2^{(p-1) / 2} /((p-1) / 2) !^{2} & \text { if } \delta=-1 .\end{cases}
$$

Thus, with the help of (3.1) we have

$$
\begin{aligned}
& (-1)^{(p+\delta) / 2} 3^{2 \delta\lfloor p / 4\rfloor}\left(\frac{\lfloor p / 4\rfloor !^{\delta}}{((p-2+\delta) / 2) ! !}\right)^{2} \\
\equiv & (-1)^{(1+\delta) / 2}\left(\frac{3}{p}\right)^{\delta}\left(\frac{2}{p}\right)^{-\delta} \delta=-\left(\frac{6}{p}\right)=(-1)^{\lfloor(p+11) / 12\rfloor}(\bmod p),
\end{aligned}
$$

and hence (1.19) follows from the above.

Case 2. $p \equiv 3(\bmod 4)$.

In this case, with the aid of (3.1) we have

$$
\begin{aligned}
& (-1)^{(p+\delta) / 2} 3^{2 \delta\lfloor p / 4\rfloor}\left(\frac{\lfloor p / 4\rfloor !^{\delta}}{((p-2+\delta) / 2) ! !}\right)^{2} \\
\equiv & (-1)^{(\delta-1) / 2} 3^{\delta((p-1) / 2-1)}\left(\frac{2^{(p-3) / 4}}{(p-1) / 2 \times\left(\begin{array}{c}
(p-3) / 2 \\
(p-3) / 4
\end{array}\right)}\right)^{2 \delta}\left(\frac{p-1}{2} !\right)^{\delta-1} \\
\equiv & \delta\left(\frac{3}{p}\right) 3^{-\delta} 2^{\delta(p+1) / 2}\left(\begin{array}{l}
(p-3) / 2 \\
(p-3) / 4
\end{array}\right)^{-2 \delta} \equiv\left(\frac{6}{p}\right) \frac{\delta 2^{\delta}}{3^{\delta}}\left(\begin{array}{l}
(p-3) / 2 \\
(p-3) / 4
\end{array}\right)^{-2 \delta}(\bmod p),
\end{aligned}
$$

and hence (1.20) holds. 
In view of the above, we have completed the proof.

Proof of Theorem 1.7. Let $n=(p-1) / 2$. Clearly,

$$
\prod_{1 \leqslant i<j \leqslant n}(j-i)=\prod_{k=1}^{n} k^{|\{1 \leqslant i \leqslant n: i+k \leqslant n\}|}=\prod_{k=1}^{n} k^{n-k}
$$

and hence

$$
\prod_{1 \leqslant i<j \leqslant n}\left(\frac{j-i}{p}\right)=\left(\frac{n !}{p}\right)^{n} \prod_{\substack{k=1 \\ 2 \nmid k}}^{n}\left(\frac{k}{p}\right) .
$$

Case 1. $p \equiv 1(\bmod 4)$.

In this case, $n$ is even, and hence by $(6.4)$ and $[15,(3.5)]$ we have

$$
\prod_{1 \leqslant i<j \leqslant n}\left(\frac{j-i}{p}\right)=\left(\frac{(n-1) ! !}{p}\right)=(-1)^{\left|\left\{0<k<\frac{p}{4}:\left(\frac{k}{p}\right)=-1\right\}\right|} .
$$

By (3.2) and [14, Lemma 2.3],

$$
\prod_{1 \leqslant i<j \leqslant n}\left(\frac{j^{2}-i^{2}}{p}\right)=\left(\frac{-n !}{p}\right)=\left(\frac{2}{p}\right)=(-1)^{(p-1) / 4} .
$$

Thus we also have

$$
\prod_{1 \leqslant i<j \leqslant n}\left(\frac{j+i}{p}\right)=(-1)^{(p-1) / 4}(-1)^{\left|\left\{0<k<\frac{p}{4}:\left(\frac{k}{p}\right)=-1\right\}\right|}=(-1)^{\left|\left\{0<k<\frac{p}{4}:\left(\frac{k}{p}\right)=1\right\}\right|} .
$$

So (1.21) holds in this case.

Case 2. $p \equiv 3(\bmod 4)$.

In this case, $n$ is odd and

$$
\prod_{1 \leqslant i<j \leqslant n}\left(\frac{j^{2}-i^{2}}{p}\right)=\left(\frac{1}{p}\right)=1
$$

by (3.2). So it suffices to prove (1.21) for $\delta=-1$.

In view of (6.4), we have

$$
\prod_{1 \leqslant i<j \leqslant n}\left(\frac{j-i}{p}\right)=\left(\frac{n !}{p}\right)\left(\frac{n ! !}{p}\right)=\left(\frac{(n-1) ! !}{p}\right) .
$$

If $p \equiv 3(\bmod 8)$, then by $[15,(3.6)]$ we have

$$
\left(\frac{(n-1) ! !}{p}\right)=(-1)^{\lfloor(p+1) / 8\rfloor}=(-1)^{(p-3) / 8}
$$

and hence (1.21) holds for $\delta=-1$. When $p \equiv 7(\bmod 8)$, we have

$$
\left(\frac{n !}{p}\right)=\left(\frac{(-1)^{(h(-p)+1) / 2}}{p}\right)=(-1)^{(h(-p)+1) / 2} \text { and }\left(\frac{n ! !}{p}\right)=(-1)^{(p+1) / 8}
$$

by Mordell [8] and Sun [15, (3.6)] respectively, hence (1.21) with $\delta=-1$ follows from (6.5).

Combining the above, we have finished the proof of (1.21). 


\section{Some Related CONJECTURES}

Motivated by our results in Section 1, here we pose 10 conjectures for further research. We have verified all the following conjectures for primes $p<13000$.

Conjecture 7.1. Let $p>3$ be a prime and let $\delta \in\{ \pm 1\}$. Then

$$
\prod_{\substack{1 \leqslant i<j \leqslant(p-1) / 2 \\ p \nmid 2 i^{2}+\delta 5 i j+2 j^{2}}}\left(\frac{2 i^{2}+\delta 5 i j+2 j^{2}}{p}\right)=\frac{1}{2}\left(\frac{\delta}{p}\right)\left(\left(\frac{-1}{p}\right)+\left(\frac{2}{p}\right)+\left(\frac{6}{p}\right)-\left(\frac{p}{3}\right)\right) .
$$

Conjecture 7.2. Let $p>3$ be a prime. Then

$$
\prod_{\substack{1 \leqslant i<j \leqslant(p-1) / 2 \\ p \nmid i^{2}-i j+j^{2}}}\left(\frac{i^{2}-i j+j^{2}}{p}\right)= \begin{cases}-1 & \text { if } p \equiv 5,7(\bmod 24), \\ 1 & \text { otherwise. }\end{cases}
$$

Also,

$$
\prod_{\substack{1 \leqslant i<j \leqslant(p-1) / 2 \\ p \nmid i^{2}+i j+j^{2}}}\left(\frac{i^{2}+i j+j^{2}}{p}\right)= \begin{cases}-1 & \text { if } p \equiv 5,11(\bmod 24), \\ 1 & \text { otherwise. }\end{cases}
$$

Conjecture 7.3. Let $p>3$ be a prime. Then

$$
\prod_{\substack{1 \leqslant i<j \leqslant(p-1) / 2 \\ p \nmid i^{2}-3 i j+j^{2}}}\left(\frac{i^{2}-3 i j+j^{2}}{p}\right)= \begin{cases}-1 & \text { if } p \equiv 7,19(\bmod 20), \\ 1 & \text { otherwise. }\end{cases}
$$

Also,

$$
\prod_{\substack{1 \leqslant i<j \leqslant(p-1) / 2 \\ p \nmid i^{2}+3 i j+j^{2}}}\left(\frac{i^{2}+3 i j+j^{2}}{p}\right)= \begin{cases}-1 & \text { if } p \equiv 19,23,27,31(\bmod 40), \\ 1 & \text { otherwise. }\end{cases}
$$

Recall that for any prime $p \equiv 3(\bmod 4)$ the class number $h(-p)$ of the imaginary quadratic field $\mathbb{Q}(\sqrt{-p})$ is odd by $[8]$.

Conjecture 7.4. Let $\delta \in\{ \pm 1\}$.

(i) For any prime $p \equiv 1(\bmod 12)$, we have

$$
T_{p}(1,4 \delta, 1) \equiv-3^{(p-1) / 4}(\bmod p) .
$$

(ii) Let $p>3$ be a prime. Then

$$
\begin{aligned}
& \prod_{\substack{1 \leqslant i<j \leqslant(p-1) / 2 \\
p \nmid i^{2}+\delta 4 i j+j^{2}}}\left(\frac{i^{2}+\delta 4 i j+j^{2}}{p}\right) \\
& = \begin{cases}1 & \text { if } p \equiv 1(\bmod 24), \\
(-1)^{\left|\left\{0<k<\frac{p}{4}:\left(\frac{k}{p}\right)=-1\right\}\right|} & \text { if } p \equiv 17(\bmod 24), \\
\delta(-1)^{\left|\left\{0<k<\frac{p}{12}:\left(\frac{k}{p}\right)=-1\right\}\right|-1} & \text { if } p \equiv 7(\bmod 24), \\
\delta(-1)^{\left|\left\{0<k<\frac{p}{12}:\left(\frac{k}{p}\right)=-1\right\}\right|+\frac{h(-p)-1}{2}} & \text { if } p \equiv 19(\bmod 24) .\end{cases}
\end{aligned}
$$

Conjecture 7.5. Let $p>3$ be a prime. Then

$$
\prod_{\substack{1 \leqslant i \leqslant j \leqslant(p-1) / 2 \\ p \nmid 4 i^{2}+j^{2}}}\left(\frac{4 i^{2}+j^{2}}{p}\right)= \begin{cases}1 & \text { if } p \equiv 1,7,9,19(\bmod 20), \\ -1 & \text { otherwise. }\end{cases}
$$


Conjecture 7.6. Let $p>3$ be a prime. Then

$$
(-1)^{\left|\left\{1 \leqslant k<p / 3:\left(\frac{k}{p}\right)=-1\right\}\right|} \prod_{\substack{i, j=1 \\ p \nmid 3 i+j}}^{(p-1) / 2}\left(\frac{3 i+j}{p}\right)= \begin{cases}1 & \text { if } p \equiv \pm 1(\bmod 12), \\ (-1)^{\lfloor p / 12\rfloor} & \text { if } p \equiv \pm 5(\bmod 12),\end{cases}
$$

and

$$
\prod_{\substack{i, j=1 \\ p \nmid 3 i-j}}^{(p-1) / 2}\left(\frac{3 i-j}{p}\right)= \begin{cases}(-1)^{\left|\left\{1 \leqslant k<p / 3:\left(\frac{k}{p}\right)=-1\right\}\right|+(p-1) / 12} & \text { if } p \equiv 1(\bmod 12), \\ (-1)^{\left|\left\{1 \leqslant k<p / 3:\left(\frac{k}{p}\right)=-1\right\}\right|-1} & \text { if } p \equiv 5(\bmod 12), \\ (-1)^{\left|\left\{1 \leqslant k<p / 6:\left(\frac{k}{p}\right)=1\right\}\right|+(p+1) / 4} & \text { if } p \equiv 7(\bmod 12), \\ -1 & \text { if } p \equiv 11(\bmod 12)\end{cases}
$$

Conjecture 7.7. Let $p>3$ be a prime. Then

$$
\prod_{\substack{i, j=1 \\ p \nmid 4 i+j}}^{(p-1) / 2}\left(\frac{4 i+j}{p}\right)= \begin{cases}1 & \text { if } p \equiv 1(\bmod 4), \\ (-1)^{(h(-p)-1) / 2+\lfloor p / 8\rfloor} & \text { if } p \equiv 3(\bmod 8), \\ (-1)^{\left|\left\{1 \leqslant k<p / 4:\left(\frac{k}{p}\right)=-1\right\}\right|} & \text { if } p \equiv 7(\bmod 8)\end{cases}
$$

and

$$
\prod_{\substack{i, j=1 \\ p \nmid 4 i-j}}^{(p-1) / 2}\left(\frac{4 i-j}{p}\right)= \begin{cases}(-1)^{(p-1) / 4} & \text { if } p \equiv 1(\bmod 4) \\ (-1)^{\lfloor p / 8\rfloor} & \text { if } p \equiv 3(\bmod 4) .\end{cases}
$$

Conjecture 7.8. Let $p>5$ be a prime. Then

$$
\begin{gathered}
(-1)^{\left|\left\{1 \leqslant k<p / 10:\left(\frac{k}{p}\right)=-1\right\}\right|} \prod_{\substack{i, j=1 \\
p \nmid 5 i+j}}^{(p-1) / 2}\left(\frac{5 i+j}{p}\right) \\
= \begin{cases}(-1)^{\lfloor(p+1) / 10\rfloor} & \text { if } p \equiv \pm 1, \pm 3(\bmod 20), \\
(-1)^{\lfloor p / 20\rfloor} & \text { if } p \equiv \pm 7(\bmod 20), \\
(-1)^{\lfloor(p+9) / 20\rfloor} & \text { if } p \equiv \pm 9(\bmod 20),\end{cases}
\end{gathered}
$$

and

$$
\prod_{\substack{i, j=1 \\ p \nmid 5 i-j}}^{(p-1) / 2}\left(\frac{5 i-j}{p}\right)= \begin{cases}(-1)^{(h(-p)+1) / 2} & \text { if } p \equiv 3,7(\bmod 20), \\ (-1)^{(p+9) / 20} & \text { if } p \equiv-9(\bmod 20), \\ (-1)^{\left|\left\{1 \leqslant k<p / 10:\left(\frac{k}{p}\right)=-1\right\}\right|+(h(-p)-1) / 2} & \text { if } p \equiv-1(\bmod 20), \\ (-1)^{\left|\left\{1 \leqslant k<p / 10:\left(\frac{k}{p}\right)=-1\right\}\right|+\lfloor(p+3) / 20\rfloor} & \text { if } p \equiv 1,-3(\bmod 20), \\ (-1)^{\left|\left\{1 \leqslant k<p / 10:\left(\frac{k}{p}\right)=-1\right\}\right|+\lfloor(p-3) / 10\rfloor} & \text { if } p \equiv-7,9(\bmod 20) .\end{cases}
$$

Conjecture 7.9. For any prime $p>3$, we have

$$
\prod_{\substack{i, j=1 \\ p \nmid 6 i+j}}^{(p-1) / 2}\left(\frac{6 i+j}{p}\right)= \begin{cases}(-1)^{\left|\left\{1 \leqslant k<p / 12:\left(\frac{k}{p}\right)=-1\right\}\right|} & \text { if } p \equiv 1(\bmod 24), \\ (-1)^{\left|\left\{\frac{p+3}{4} \leqslant k \leqslant\left\lfloor\frac{p+1}{3}\right\rfloor:\left(\frac{k}{p}\right)=-1\right\}\right|} & \text { if } p \equiv 5,-7,-11(\bmod 24), \\ (-1)^{(h(-p)+1) / 2+\lfloor(p+1) / 24\rfloor} & \text { if } p \equiv-1,-5(\bmod 24), \\ (-1)^{\lfloor p / 24\rfloor-1} & \text { if } p \equiv 7,11(\bmod 24),\end{cases}
$$

and

$$
\prod_{\substack{i, j=1 \\ p \nmid 6 i-j}}^{(p-1) / 2}\left(\frac{6 i-j}{p}\right)=(-1)^{\left|\left\{\frac{p+2}{4}<k<\frac{p}{3}:\left(\frac{k}{p}\right)=1\right\}\right|} .
$$


Conjecture 7.10. Let $p>3$ be a prime. Then

$$
(-1)^{\left|\left\{1 \leqslant k<p / 4:\left(\frac{k}{p}\right)=-1\right\}\right|} \prod_{\substack{i, j=1 \\ p \nmid 8+j}}^{(p-1) / 2}\left(\frac{8 i+j}{p}\right)= \begin{cases}(-1)^{(p+1) / 8} & \text { if } p \equiv-1(\bmod 8), \\ 1 & \text { otherwise, }\end{cases}
$$

and

$$
\prod_{\substack{i, j=1 \\ p \nmid 8 i-j}}^{(p-1) / 2}\left(\frac{8 i-j}{p}\right)= \begin{cases}(-1)^{\left|\left\{1 \leqslant k<p / 4:\left(\frac{k}{p}\right)=1\right\}\right|} & \text { if } p \equiv 1(\bmod 4) \\ (-1)^{(h(-p)+1) / 2+(p-3) / 8} & \text { if } p \equiv 3(\bmod 8) \\ -1 & \text { if } p \equiv 7(\bmod 8)\end{cases}
$$

Acknowledgments. The research is supported by the Natural Science Foundation of China (Grant No. 11971222). The author would like to thank the the referee for helpful comments.

\section{REFERENCES}

[1] B. C. Berndt, R. J. Evans and K. S. Williams, Gauss and Jacobi Sums, John Wiley \& Sons, 1998.

[2] K. Burde, Eine Verteilungseigenschaft der Legendresymbole, J. Number Theory 12 (1980), 273-277.

[3] S. Chowla, B. Dwork and R. J. Evans, On the $\bmod p^{2}$ determination of $\left(\begin{array}{c}(p-1) / 2 \\ (p-1) / 4\end{array}\right)$, J. Number Theory 24 (1986), 188-196.

[4] L. E. Dickson, History of the Theory of Numbers, Vol. III, AMS Chelsea Publ., 1999.

[5] R. H. Hudson and K. S. Williams, Class number formulae of Dirichlet type, Math. Comp. 39 (1982), 725-732.

[6] K. Ireland and M. Rosen, A Classical Introduction to Modern Number Theory, 2nd Edition, Graduate Texts in Math., 84, Springer, New York, 1990.

[7] M. Jenkins, Proof of an Arithmetical Theorem leading, by means of Gauss fourth demonstration of Legendres law of reciprocity, to the extension of that law, Proc. London Math. Soc. 2 (1867) 29-32.

[8] L. J. Mordell, The congruence $((p-1) / 2) ! \equiv \pm 1(\bmod p)$, Amer. Math. Monthly 68 (1961) $145-146$.

[9] H. Rademacher, Lectures on Elementary Number Theory, Blaisdell Publishing Company, New York, 1964.

[10] P. Ribenboim, The Book of Prime Number Records, Springer, New York, 1980.

[11] Z.-H. Sun, Values of Lucas sequences modulo primes, Rocky Mountain J. Math. 33 (2013) $1123-1145$.

[12] Z.-H. Sun and Z.-W. Sun, Fibonacci numbers and Fermat's last theorem, Acta Arith. 60 (1992) 371-388.

[13] Z.-W. Sun, Binomial coefficients, Catalan numbers and Lucas quotients, Sci. China Math. 53 (2010) 2473-3488.

[14] Z.-W. Sun, On some determinants with Legendre symbol entries, Finite Fields Appl. 56 (2019) 285-307.

[15] Z.-W. Sun, Quadratic residues and related permutations and identities, Finite Fields Appl. 59 (2019) 246-283.

[16] K. S. Williams, On the quadratic residues $(\bmod p)$ in the interval $(0, p / 4)$, Canad. Math. Bull. 26 (1983) 123-124.

[17] K. S. Williams and J. D. Currie, Class numbers and biquadratic reciprocity, Canad. J. Math. 34 (1982) 969-988.

Department of Mathematics, Nanjing University, Nanjing 210093, People's Republic OF CHINA

E-mail address: zwsun@nju.edu.cn 